\title{
Wechselbeziehungen zwischen dem Milchleistungsniveau, der Fruchtbarkeit und dem Krankheitsgeschehen bei Holstein Friesian Milchkühen
}

\author{
HEIKO SCHOLZ' ${ }^{1}$ BIRGIT BEYER' ${ }^{1}$, GERHARD ANACKER ${ }^{2}$ und MARTIN WÄHNER ${ }^{1}$
}

'Fachbereich Landwirtschaft, Ökotrophologie und Landschaftsentwicklung, Hochschule Anhalt (FH), Bernburg, Deutschland ${ }^{2}$ Thüringer Landesanstalt für Landwirtschaft, Jena, Deutschland

\section{Zusammenfassung}

In einem Thüringer Holstein-Friesian-Zuchtbetrieb wurden über insgesamt 5 Jahre die Daten von 1841 Laktationen von 353 Kühen analysiert sowie die Daten der Fruchtbarkeit und das Auftreten von Erkrankungen erfasst. Es konnte beobachtet werden, dass der geringste Erstbesamungserfolg mit $40 \%$ bei einer Laktationsleistung zwischen $9000 \mathrm{~kg}$ und $11000 \mathrm{~kg}$ lag. Die Rastzeit der Kühe variierte in Abhängigkeit von der Art und dem Zeitpunkt der ersten Erkrankung in der Laktation. Mit steigender Laktationsleistung der Kühe konnte eine tendenzielle Verminderung der Erkrankungshäufigkeit und des Erstbesamungserfolges dokumentiert werden. So wiesen die Kühe mit einer 305-TageMilchleistung von mehr als $11000 \mathrm{~kg}$ mit $56 \%$ die niedrigste Erkrankungshäufigkeit auf. Im Mittel wurden 60\% der Kühe mindestens einmal im Laufe einer Laktation behandelt. Die häufigsten Erkrankungen betrafen die Fruchtbarkeit und die Eutergesundheit. Bei den Jungkühen konnte eine Verminderung des Erstbesamungserfolges bei steigender Erkrankungshäufigkeit beobachtet werden.

Schlüsselwörter: Rind, Milchleistung, Fruchtbarkeit, Krankheitsgeschehen, Holstein Friesian

\section{Abstract}

\section{Relationship between milk yield, fertility and amount of disease of Holstein Friesian dairy cows}

Data of 1841 lactations of 353 Holstein Friesian dairy cows and data according to their reproductive fertility and diseases were analysed over a period of five years in a breeding farm in Thüringen (Germany). As a result the lowest success of first service has been achieved at a lactation (ranged) between 9000 and $11000 \mathrm{~kg}$. The time between calving and the following first service varied according to the first disease in last lactation. With rising milk yield the frequency of diseases and the success of first service tend to decrease. Cows achieving a 305 day milk yield of more than $11000 \mathrm{~kg}$ showed with $56 \%$ the lowest frequency of diseases. In average $60 \%$ of all dairy cows need to be treated once in a lactation. The diseases of highest prevalence are in the field of reproductive fertility and udder health. For heifers a decreased success of first service has been detected if they showed an increased prevalence of illness.

Keywords: cattle, lactation, fertility, illness, Holstein Friesian 


\section{Einleitung}

Eine hohe Milchleistung ist für die wirtschaftliche Gestaltung des Produktionszweiges Milchproduktion eine wesentliche Voraussetzung. Jedoch kann mit der Erhöhung der Leistung der Milchkühe auch eine Erhöhung der Reproduktionsraten und eine Verschlechterung der Fruchtbarkeitsergebnisse in der Gesamtpopulation beobachtet werden (ADR 2007, BERGFELD und KLUNKER 2002, LUCY 2001, SEELAND und HENZE 2003, TLL 2008). Ein vermehrtes Auftreten von Krankheiten kann einen Abfall der Nutzungsdauer und einen Anstieg der Zwangsmerzungsrate nach sich ziehen. Langfristig sind daher funktionale Merkmale in die Zuchtarbeit einzubeziehen (AMIN 2000, 2001, ATIL 2000, DISTL 2001, HINRICHS et al. 2006, LAZAREVIC und MISCEVIC 2005, SWALVE 2003). Durch die relativ geringe genetische Beeinflussung der Reproduktionsmerkmale im Vergleich zu den managementseitig beeinflussbaren Umwelteinflüssen können jedoch Probleme mit der Fruchtbarkeit der Tiere in sehr gut geführten Unternehmen vermindert werden. Bei leistungsgerechter Fütterung und Haltung können sich nach SPAIN (2005) Leistung und Tiergesundheit jedoch synergetisch ergänzen. Diesen Zusammenhang bestätigen auch die Ergebnisse anderer Untersuchungen (DUNKLEE et al. 1994, LUCY 2001). Auch SCHRÖDER und STAUFENBIEL (2003) sowie BIELFELD et al. (2004) zeigen auf, dass ein effizientes Herdenmanagement die Fruchtbarkeitsprobleme in gewissen Grenzen ausgleichen kann. Ergebnisse von VARNER (2002) belegen, dass bis zu $60 \%$ der problematisch erscheinenden Kühe im Bereich der Fruchtbarkeit organisch gesund sind, aufgrund verschiedener Einflüsse jedoch vom Stallpersonal nicht als brünstig erkannt werden. Aber auch Stress beeinflusst die Fruchtbarkeitsleistung der Tiere negativ (MANTEUFFEL 2002). Es bleibt auch weiterhin eine Herausforderung, eine hohe Milchleistung und eine gute Fruchtbarkeit bei den Hochleistungstieren zu erzielen, die sich mit dem sinkenden mittleren Herdendurchschnittsalter verschärft (SPIEKERS und POTTHAST 2004). Für die Züchtung bedeutungsvoll bleibt jedoch der Antagonismus zwischen Milchleistung und Fruchtbarkeitsleistung (SWALVE 2003). So konnte BUTLER (2003) aufzeigen, dass bei Milchkühen in den USA mit der Erhöhung der Milchleistung eine Verschlechterung des Besamungserfolges einherging. Weitere Untersuchungen belegen diesen Zusammenhang (AMIN 2001, ANACKER 2002, BERGFELD und KLUNKER 2002, OSCHIKA 2002). Die Verschlechterungen der Fruchtbarkeit im Milchviehbestand müssen aber nicht mit einer Abnahme der Fruchtbarkeit im Färsenbestand einhergehen, was die Ergebnisse von BOUSQUET et al. (2004), PLATEN et al. (1999) sowie ROYAL et al. (2000) belegen. Ziel der vorliegenden Untersuchung war es, die Beziehungen zwischen der Milchleistung der Kühe, deren Fruchtbarkeit sowie dem Auftreten von Krankheiten zu analysieren.

\section{Material und Methoden}

Die Datengrundlage bildeten die Erhebungen in einem Thüringer Holstein-FriesianZuchtbetrieb über insgesamt 5 Jahre, in denen insgesamt 1841 Laktationen von 353 Kühen zur Verfügung standen. Das landwirtschaftliche Unternehmen befindet sich in den Übergangslagen der Mittelgebirge. Alle Kühe wurden entsprechend ihres Reproduktionsoder Laktationsstadiums gruppenweise in Laufställen mit Tiefliegeboxen und 
planbefestigten Laufgängen gehalten. Die Fütterung erfolgte über eine Totale Mischration für die einzelnen Leistungsgruppen, wobei das Grobfutter aus dem eigenen Anbau stammte. Mit einem 22er Melkkarussell erfolgte dreimal täglich der Milchentzug. Die mittlere Milchleistung je Kuh und Jahr betrug im Jahr 2007 etwa 10900 kg (TVL 2008). Im Mittel aller Betriebe Thüringens lag das Niveau der Milchleistungsprüfung bei $8633 \mathrm{~kg}$ mit 4,08\% Fett und 3,39\% Eiweiß (TVL 2008), die mittlere Milchleistung der Kontrollverbände der Neuen Bundesländer lag bei $8675 \mathrm{~kg}$ mit 4,09\% Fett und 3,40\% Eiweiß (VIT 2008).

Die 305-Tage-Milchleistung der Kühe wurde für alle Auswertungen herangezogen, wobei alle abgeschlossenen Laktationen mit weniger als 280 Melktagen für die Berechnungen eliminiert wurden. Die Gruppeneinteilung sowohl für die 100-Tage-Milchleistung als auch die 305-Tage-Milchleistung ist in Tabelle 1 enthalten.

Tabelle 1

Gruppeneinteilung für die 100- und 305-Tage-Milchleistung

Classification for 100-and 305-day-milk yield

\begin{tabular}{lcc}
\hline Gruppe & 100 -Tage-Milchleistung & 305-Tage-Milchleistung \\
\hline 1 & $<3300 \mathrm{~kg}$ & $<9000 \mathrm{~kg}$ \\
2 & 3300 bis unter $4100 \mathrm{~kg}$ & 9000 bis unter $11000 \mathrm{~kg}$ \\
3 & $\geq 4100 \mathrm{~kg}$ & $\geq 11000 \mathrm{~kg}$ \\
\hline
\end{tabular}

Neben den Daten zur Milchleistung und den Milchinhaltsstoffen der Kühe in den entsprechenden Laktationen wurden unter anderem folgende Kennzahlen im Unternehmen erfasst:

- Erkrankung des Tieres mit einer genauen Zuordnung wie Nachgeburtsverhaltung, Festliegen, Fruchtbarkeit, Euter, Klauen und Gliedmaßen, Stoffwechsel, sonstige Erkrankungen,

- Anzahl der Erkrankungen in der aktuellen Laktation,

- bakteriologische Befunde der Einzeltiere,

- Abgangsursache aus dem Bestand,

- Trächtigkeitsbefunde.

Die Stammdaten der Kühe (u. a. Alter, Abstammung, Erstkalbealter, Laktationsbeginn und Laktationsende) sowie die Brunst- und Besamungsdaten wurden dem Herdenmanagementprogramm SUPERKUH (Firma agrocom) entnommen und den entsprechenden Tieren zugeordnet. Die Kennzahlen Zwischentragezeit, Zwischenkalbezeit und Rastzeit wurden berechnet.

In Tabelle 2 wird die Einteilung der Gruppen für die Merkmale Laktationsnummer, Anzahl Besamungen, Erstkalbealter und Zwischentragezeit ersichtlich. Es ist dabei zu beachten, dass sich die gebildeten Gruppen für das Erstkalbealter und die Zwischentragezeit auf den gesamten Untersuchungszeitraum beziehen und somit zum Beispiel mögliche jährliche Schwankungen aufgrund betrieblicher Gegebenheiten nicht berücksichtigt wurden. 
Tabelle 2

Gruppeneinteilung für die Merkmale Laktationsnummer, Anzahl Besamungen, Erstkalbealter und Zwischentragezeit

classification of number of lactation, number of insemination, age of first calving, days open

\begin{tabular}{lcccc}
\hline Gruppe & Laktationsnummer & Anzahl Besamungen & Erstkalbealter, Monate & Zwischentragezeit, $d$ \\
\hline 1 & 1 & 1 & $<26$ & $<104$ \\
2 & $2-3$ & $2-3$ & $26-30$ & $104-181$ \\
3 & $>3$ & $>3$ & $\geq 30$ & $\geq 181$ \\
\hline
\end{tabular}

Für die statistischen Auswertungen wurde eine Klasseneinteilung auf der Grundlage des Mittelwertes und einer halben Standardabweichung gewählt $(x \pm 1 / 2 s)$. Die Klassen 1 und 3 wurden dabei immer als offene Klassen definiert. Die Datenauswertung wurde mit der PC-Version des Statistikprogrammpaketes SPSS 14.0 (SPSS 2005) und SAS (SAS 2006) vorgenommen. Die Korrelationskoeffizienten wurden nach den Methoden von Pearson und Spearman berechnet. Auftretende Mittelwertdifferenzen wurden bei einer Irrtumswahrscheinlichkeit von $P \leq 0,05$ auf Signifikanz überprüft. In den Tabellen und Abbildungen erscheinen die Signifikanzsymbole als Exponenten.

\section{Ergebnisse und Diskussion}

Die durchschnittliche Milchmenge aller in die Untersuchungen einbezogenen Kühe betrug $10213 \mathrm{~kg} \pm 1900 \mathrm{~kg}$ und die Tiere erreichten eine mittlere Anzahl an Laktationen von 2,4. Es konnte eine Zwischentragezeit von $142 \pm 78$ Tagen bei einem Besamungsaufwand von durchschnittlich 2,0 dokumentiert werden. Der Erstbesamungserfolg im Untersuchungszeitraum betrug 50\%. Nach DE KRUIF et al. (1998) sollten in Betrieben mit guter Herdenfruchtbarkeit $75 \%$ der Tiere eine Zwischentragezeit von weniger als 115 Tagen aufweisen und mindestens $60 \%$ Erstbesamungserfolg aufweisen. Kennzahlen der Fruchtbarkeitsleistung sollten aus Sicht der Verfasser im Zusammenhang beurteilt werden.

In den Untersuchungen konnte der niedrigste Wert für den Erstbesamungserfolg in der mittleren Leistungsklasse sowohl für die 100-Tage- als auch die 305-TageMilchleistung dokumentiert werden (Tabelle 3). Dies trifft sowohl für die Jung- als auch die Kühe ab der 2. Laktation zu. Bei den Tieren mit einer 100-Tage-Leistung konnte weiterhin beobachtet werden, dass die Jungkühe mit durchschnittlich $52 \%$ einen höheren Trächtigkeitserfolg aus der Erstbesamung aufwiesen als die Kühe ab der 2. Laktation (48\%). Die Differenz konnte jedoch nicht statistisch gesichert werden. Für Kühe mit einer 305-Tage-Laktation ergeben sich geringere Unterschiede, wobei die Jungkühe einen Erstbesamungserfolg von $46 \%$ und die Kühe ab der 2. Laktation von $44 \%$ aufwiesen. Auch in den Untersuchungen von SEELAND und HENZE (2003) sowie VOGEL (2004) wiesen die Jungkühe einen höheren Erstbesamungserfolg als die Altkühe auf.

Die mittlere Rastzeit der Kühe in den eigenen Untersuchungen betrug $102 \pm 53$ Tage. HEUWIESER (2002) empfiehlt für Hochleistungskühe eine Rastzeit zwischen 60 und 70 Tagen, was durch Ergebnisse anderer Untersuchungen untermauert wurde (BUCHWALD und PIEPER 2001, JAHNKE 2002). FÜRST et al. (2007) zeigen auf, dass sich ab dem 50. Laktationstag eine Verlängerung der Rastzeit unwesentlich auf die Fruchtbarkeitsergebnisse auswirkt. In der Literatur wird immer wieder der Zusammenhang zwischen 
einer hohen Milchleistung und einer erhöhten Rastzeit dieser Tiere diskutiert, wobei die Fütterung und die Umweltbedingungen beachtet werden müssen (ATIL 2000, GRUNERT 1996). Für die vorliegenden Daten konnte kein signifikanter Zusammenhang zwischen beiden Kennzahlen errechnet werden, wobei die längste Rastzeit bei den Jungkühen mit sehr hohen Milchleistungen auftrat (Tabelle 4). Ergebnisse von STAUFENBIEL et al. (2003) zeigen aber auch, dass in Betrieben mit hohen Laktationsleistungen Rastzeiten unter 80 Tagen möglich sind.

Tabelle 3

Beziehungen zwischen der mittleren 100-Tage- bzw. 305-Tage-Milchleistung und dem Erstbesamungserfolg Relationship between average 100-and 305-day-milk yield and success of first insemination

\begin{tabular}{lcc}
\hline & \multicolumn{2}{c}{$\begin{array}{c}\text { Erstbesamungserfolg } \\
\text { Kühe ab 2. Laktation }\end{array}$} \\
\hline 100-Tage-Milchleistung & & \\
$<3300 \mathrm{~kg}$ & $55 \%$ & $56^{\mathrm{a}} \%$ \\
$3300 \mathrm{bis}$ unter $4100 \mathrm{~kg}$ & $48 \%$ & $46^{\mathrm{b}} \%$ \\
$>4100 \mathrm{~kg}$ & $55 \%$ & $50 \%$ \\
$305-$ Tage-Milchleistung & & \\
$<9000 \mathrm{~kg}$ & $52 \%$ & $54^{\mathrm{a}} \%$ \\
9000 bis unter $11000 \mathrm{~kg}$ & $39^{\mathrm{a}} \%$ & $40^{\mathrm{b}} \%$ \\
$>11000 \mathrm{~kg}$ & $57^{\mathrm{b}} \%$ & $47 \%$ \\
\hline
\end{tabular}

a,b Werte innerhalb einer Spalte mit verschiedenen Buchstaben sind signifikant verschieden $(P<0,05)$.

Tabelle 4

Einfluss der 305-Tage-Milchleistung auf die Rastzeit in Abhängigkeit vom Alter der Milchkühe Influence of 305-day-milk yield to first service interval in depence of age of dairy cows

\begin{tabular}{lccc}
\hline Milchleistung & alle Kühe & $\begin{array}{c}\text { Rastzeit, Tage } \\
\text { Jungkühe }\end{array}$ & Kühe ab 2. Laktation \\
\hline$<9000 \mathrm{~kg}$ & $114 \pm 70$ & $113 \pm 71$ & $114 \pm 67$ \\
$9000 \mathrm{bis}$ unter $11000 \mathrm{~kg}$ & $103 \pm 51$ & $104 \pm 53$ & $101 \pm 47$ \\
$>11000 \mathrm{~kg}$ & $113 \pm 47$ & $127 \pm 52$ & $106 \pm 42$ \\
\hline
\end{tabular}

Der Korrelationskoeffizient zwischen der Rastzeit und der 305-Tage-Milchleistung betrug $r=0,002(P=0,968)$. In den Untersuchungen von ZELFEL (2008) war die Laktationsleistung mit der Rastzeit ebenfalls wenig korreliert $(r=0,11)$. Dies zeigt, dass die Rastzeit der Milchkühe in den vorliegenden Untersuchungen mit der Höhe der Einsatzleistung nicht im Zusammenhang stand. Es konnte in den vorliegenden Untersuchungen ein Korrelationskoeffizient von $\mathrm{r}=-0,008 \quad(P=0,847)$ zwischen Rastzeit und der 100-TageMilchleistung dokumentiert werden.

Die Rastzeit variiert in Abhängigkeit von der Art der ersten Erkrankung nach der Abkalbung (Abbildung 1). Kühe, die innerhalb der Laktation generell nicht erkrankten, wiesen sowohl bei der Rastzeit mit 93 Tagen als auch bei der Zwischentragezeit mit 128 Tagen die geringsten Werte auf. Die höchsten Werte bei der Rastzeit und der Zwischentragezeit mussten bei Kühen mit Erkrankungen der Klauen und Gliedmaßen beobachtet werden. 


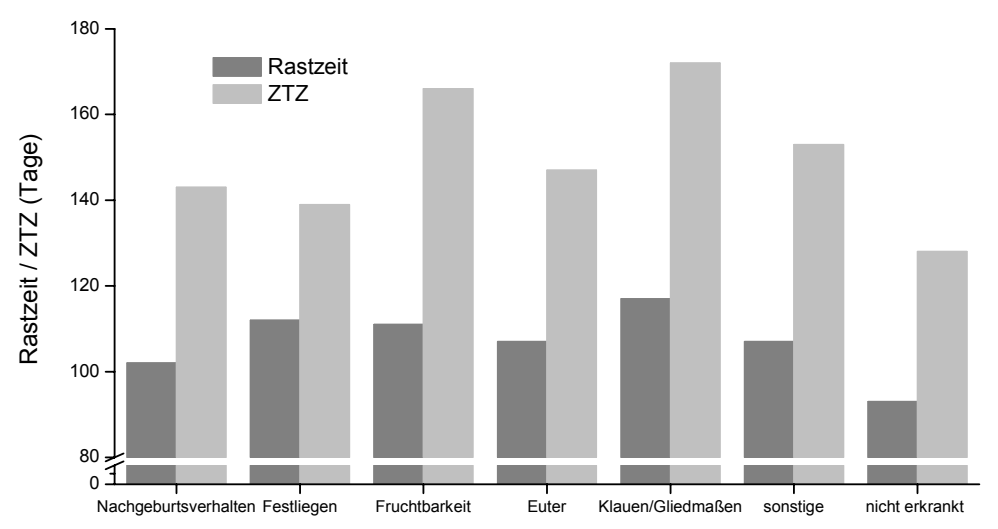

Abbildung 1

Rastzeit und Zwischentragezeit der Kühe in Abhängigkeit von der Art der Erkrankung

Days to first service and days open of cows in depence of disease

Die Zwischentragezeit wird durch den Erstbesamungserfolg beeinflusst. Die Kühe, die nach der ersten Besamung tragend waren, besaßen eine mittlere Zwischentragezeit von $106 \pm 56$ Tagen, die sich signifikant von der der übrigen Kühe, die eine Zwischentragezeit von $182 \pm 82$ Tage hatten, unterschied. Mit steigendem Besamungsaufwand konnte ebenfalls eine Verlängerung der Zwischentragezeit dokumentiert werden (Abbildung 2). Auffallend ist hier der progressive Anstieg der Zwischentragezeit. Kühe, die mit der 2. oder 3. Besamung tragend wurden, weisen im Mittel eine Zwischentragezeit von 159 Tagen auf und liegen somit 53 Tage über dem Mittel der Kühe, die aus der Erstbesamung tragend wurden. Es konnte weiterhin beobachtet werden, dass die Kühe mit mehr als einer Besamung im Mittel eine signifikant geringere Rastzeit aufwiesen als die Tiere, die mit der Erstbesamung tragend wurden.

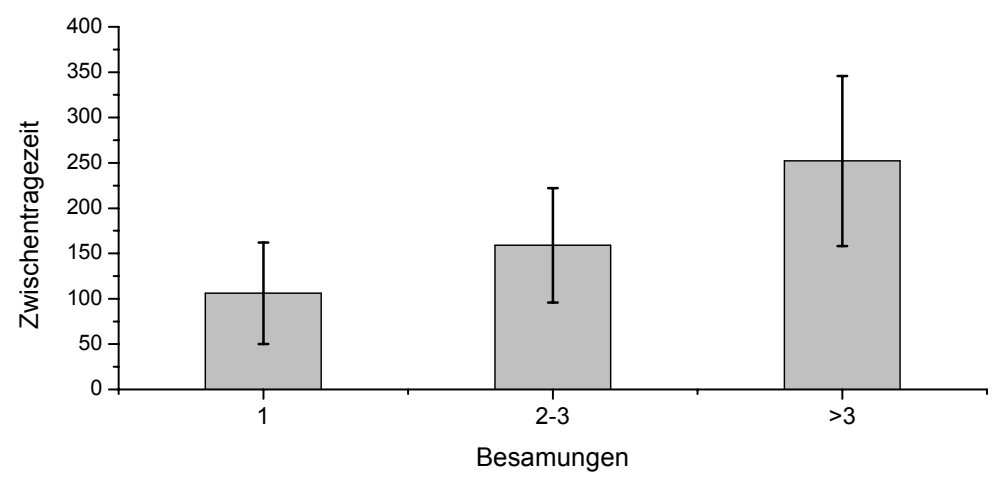

Abbildung 2

Zusammenhang zwischen der Anzahl Besamungen je Trächtigkeit und der mittleren Zwischentragezeit Relationship between number of insemination and average days open 
WANGLER und HARMS (2006) zeigen, dass Kühe mit hohen Laktationsleistungen nicht häufiger erkrankten als Kühe mit geringeren Leistungen. TENHAGEN et al. (2005) leiten aus ihren Untersuchungen die Schlussfolgerung ab, dass eine hohe Milchleistung allein kein Beleg für Gesundheit ist, dass es aber eine enge Beziehung zwischen Gesundheit und Konzeptionsbereitschaft gibt. In den vorliegenden Untersuchungen zeigte sich, dass mit steigender 305-Tage-Milchleistung eine tendenziell abnehmende Häufigkeit des Auftretens von Erkrankungen sowie eine Verminderung des Erstbesamungserfolges zu verzeichnen ist (Abbildung 3). So konnte bei den Kühen mit mehr als $11000 \mathrm{~kg}$ Milch je Jahr mit $56 \%$ die geringste Erkrankungshäufigkeit dokumentiert werden.

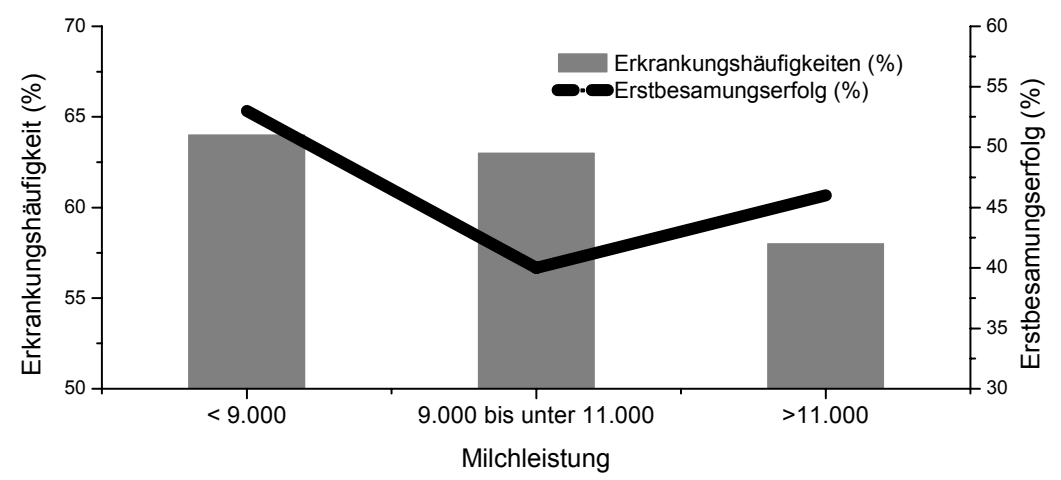

Abbildung 3

Zusammenhang zwischen der Häufigkeit des Auftretens von Erkrankungen und der 305-TageMilchleistung

Relationship between frequency of disease and 305-day-milk yield

In den vorliegenden Untersuchungen konnte mit steigender Erkrankungshäufigkeit eine Verminderung der Laktationsleistung dokumentiert werden (Abbildung 4). Vorangegangene Untersuchungen bestätigen diesen Zusammenhang (ANACKER 2003, GEIGER 2006).

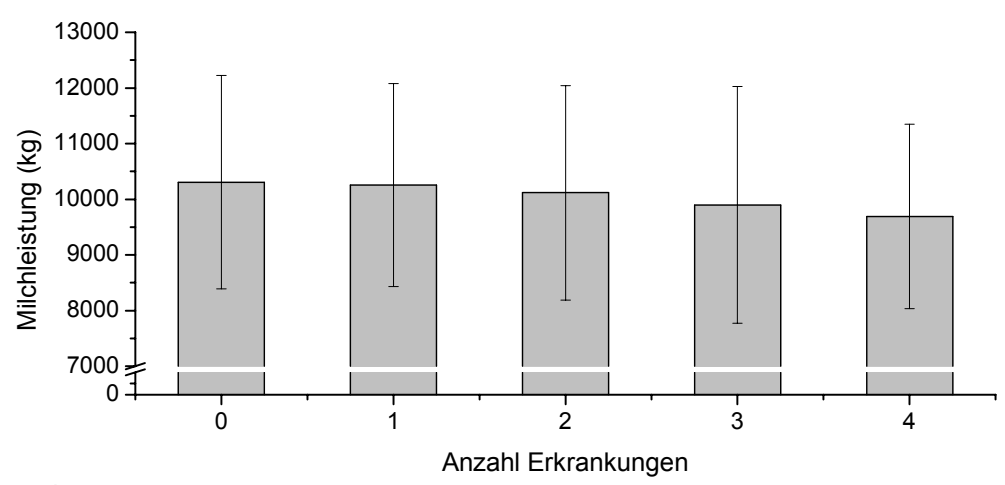

Abbildung 4

Mittlere Milchleistung der Kühe aller Laktationen in Abhängigkeit von der Erkrankungshäufigkeit Average milk yield of cows in depence of frequency of disease 
Innerhalb einer Laktation wurden alle Erkrankungen erfasst, wobei das Maximum bei 4 Erkrankungen lag. Durchschnittlich erkrankten 59,7\% aller Kühe mindestens einmal. Weiterhin wurden 28,5\% aller Kühe ein zweites Mal behandelt, bei 10,7\% bzw. 2,1\% traten eine 3. bzw. 4. Erkrankung auf. Dabei standen, wie u.a. von DISTL (2001) und HINRICHS et al. (2006) berichtet, Fruchtbarkeits- und Eutererkrankungen im Vordergrund, gefolgt von Nachgeburtsverhaltungen und Erkrankungen der Klauen und Gliedmaßen.

Verschiedene Untersuchungen belegen, dass Krankheiten (LUCY 2001, BOUSQUET et al. 2004) und die negative Energiebilanz der Kühe mehr Einfluss auf die Fruchtbarkeit besitzen als die Milchleistung. Es konnte beobachtet werden, dass die Häufigkeit der Erkrankungen der Kühe in der Laktation nur einen geringen Einfluss auf die 305-TageMilchleistung ausübte (Tabelle 5). Der Erfolg der Erstbesamung scheint bei den Jungkühen einem Einfluss durch die Häufigkeit der Erkrankungen zu unterliegen. Bei den Kühen ab der 2. Laktation konnte kein Zusammenhang zwischen beiden Kennzahlen beobachtet werden (Tabelle 5).

Tabelle 5

Einfluss der Erkrankungshäufigkeit während der Laktation auf den Erstbesamungserfolg und die 305Tage-Milchleistung in Abhängigkeit vom Alter der Kühe

Effect of frequency of disease during lactation of success of first insemination and 305-day-milk yield in depence of age of cows

\begin{tabular}{lcccc}
\hline $\begin{array}{l}\text { Erkrankungs- } \\
\text { häufigkeit }\end{array}$ & Erstbesamungserfolg & $\begin{array}{c}\text { J05-Tage- } \\
\text { Milchleistung, kg }\end{array}$ & Erstbesamungserfolg & $\begin{array}{c}\text { Kühe ab 2. Laktation } \\
\text { 305-Tage- } \\
\text { Milchleistung, kg }\end{array}$ \\
\hline 0 & $55^{\mathrm{a}} \%$ & $9427 \pm 1924$ & $56 \%$ & $10760 \pm 1814$ \\
1 & $52 \%$ & $9463 \pm 1782$ & $39 \%$ & $10655 \pm 1865$ \\
2 & $49 \%$ & $9564 \pm 1873$ & $48 \%$ & $10676 \pm 1820$ \\
3 & $21^{\mathrm{b}} \%$ & $9465 \pm 1953$ & $45 \%$ & $10121 \pm 2541$ \\
\hline
\end{tabular}

a,b Werte innerhalb einer Spalte mit verschiedenen Buchstaben sind signifikant verschieden $(P<0,05)$.

Jungkühe, die in der ersten Laktation dreimal erkrankten, wiesen mit durchschnittlich $21 \%$ den geringsten Erstbesamungserfolg auf. Die Milchleistung dieser Tiere wurde jedoch nicht durch die Häufigkeit der Erkrankungen beeinflusst. Dagegen belegen Ergebnisse von TENHAGEN et al. (2005) eine Beziehung zwischen Milchleistung und Fruchtbarkeit bei den Jungkühen, wobei der Einfluss der Leistung bei den älteren Kühen hinter den Effekt der Gesundheitsstörungen zurücktritt.

Aus den vorliegenden Ergebnissen lassen sich folgende Schlussfolgerungen ableiten:

- Wechselbeziehungen zwischen der Milchleistung der Kühe und dem Erstbesamungserfolg, der Rast- und Zwischentragezeit konnten nicht nachgewiesen werden. Daraus kann geschlussfolgert werden, dass die Kennzahlen der Fruchtbarkeit im Milchviehbestand vom Fruchtbarkeitsmanagement mehr beeinflusst werden können als von der Milchleistung der Kühe.

- Der Erstbesamungserfolg wurde durch die Milchleistung der Kühe signifikant beeinflusst, wobei die schlechtesten Ergebnisse in der mittleren Leistungsklasse (9000 bis unter $11000 \mathrm{~kg}$ je Jahr) beobachtet wurden. Eine pauschale Festlegung bestimmter Rastzeiten in Abhängigkeit von der Höhe der Milchleistung scheint für das Erzielen hoher Fruchtbarkeitskennzahlen nicht geeignet zu sein. 
- Zwischen der Höhe der 305-Tage-Laktationsleistung und dem Auftreten von Erkrankungen konnte kein signifikanter Zusammenhang ermittelt werden. Es zeigte sich, dass die Kühe ab 11000 kg Milch je Laktation mit $56 \%$ die geringste Erkrankungsrate aufwiesen. Das Erreichen hoher Milchleistungen bei einer stabilen Gesundheit stellt also keinen Widerspruch dar.

- Mit zunehmender Häufigkeit der Erkrankungen innerhalb einer Laktation konnte eine tendenzielle Erhöhung des Besamungsaufwandes beobachtet werden, wobei keine Auswirkungen auf die Höhe der 305-Tage-Milchleistung nachzuweisen waren.

\section{Literatur}

ADR (2007) Rinderproduktion in Deutschland 2006; ADR, Bonn, Deutschland

Amin AA, Gere T, Kishk WH (2000) Additive genetic variance and covariance in some reproductive disorders in Hungarian Holstein Friesian using multi-trait animal model. Arch Tierz 43, 573-81

Amin AA (2001) Lactation and sample test-day multi-trait animal model for genetic evaluation of somatic cell scores in Hungarian Holstein Friesian crossbreeds. Arch Tierz 44, 263-75

Anacker G (2002) Einfluss von Züchtung und Management auf die Tiergesundheit. Vortrag zur 7. Thüringer Ökolandbau-Fachtagung, Vachdorf, Deutschland

Anacker G (2003) Hochleistung und Tiergesundheit bei Milchkühen. Arch Tierz 46, 57-62

Atil H (2000) Genetic relationship between days open and days dry with milk yield in a herd of Holstein Friesian cattle; Arch Tierz 43, 583-90

Bergfeld U, Klunker M (2002) Bedeutung funktionaler Merkmale in der Rinderzucht und Möglichkeiten für deren züchterische Verbesserung. Arch Tierz 45 Sonderheft, 60-7

Bielfeld JC, Badertscher R, Tölle K-H, Krieter J (2004) Influence of systematic effects on fertility traits in Swiss Brown cows. Arch Tierz 47, 537-49

Bousquet D, Bouchard É, du Tremblay D (2004) Decreasing Fertility in Dairy Cows: Myth or Reality?; Proceedings of the WBC Congress, Quebec, Kanada

Buchwald K, Pieper B (2001) Fruchtbarkeitsmanagement und Ergebnisse der Dabergotzer Agrar GmbH. Workshop 2001, Dr. Pieper Technologie- und Produktentwicklung GmbH Neuruppin, Deutschland, 60-8

Butler WR (2003) Energy balance relationships with follicular development, ovulation and fertility in postpartum dairy cows. Livest Prod Sci 83, 211-8

de Kruif A, Mansfeld R, Hoedemaker M (1998) Milchleistung und Fütterung; Tierärztliche Bestands betreuung beim Milchrind, Enke Verlag, Stuttgart, Deutschland

Distl $O$ (2001) Die Bedeutung von Gesundheitsmerkmalen in der Zucht von Milchrindern. Arch Tierz 44, 365-80

Dunklee JS, Freeman AE, Kelley DH (1994) Comparison of Holsteins selected for high and average milk production. 2. Health and reproductive response to selection for milk. J Dairy Sci 77, 3683-90

Fürst C, Emmerling E, Dodenhoff J, Krogmeier D, Niebel E (2007) Zuchtwertschätzung beim Rind Grundlagen, Methoden, Modelle. ZuchtData EDV-Dienstleistungen GmbH, Wien, Österreich

Geiger H (2006) Untersuchungen zum Einfluss eines erhöhten Energie- und/oder Proteingehaltes in der Vorbereitungsfütterung auf die Milchleistung, Fruchtbarkeit, Gesundheit und Überlebensrate von Jungkühen im Verlauf der ersten Laktation. Dissertation, Freie Universität Berlin, Deutschland

Grunert E (1996) Buiatrik, Bd. 1, 5. Aufl. Verlag M \& H Schaper Alfeld, Hannover, Deutschland

Heuwieser W (2002) Verbesserung der Fruchtbarkeit bei Kühen mit hohen Leistungen. Tagungsbericht zum 6. Symposium, Dr. Pieper Technologie- und Produktentwicklung GmbH Neuruppin, 63-72

Hinrichs D, Stamer E, Junge W, Kalm E (2006) Genetic analysis of several disease categories using test day threshold models in German Holstein cows. Arch Tierz 49, 3-16

Jahnke B (2002) In jedem Jahr ein Kalb? Neue Landw 13 Sonderheft Rind, 22-5

Lazarevic R, Miscevic B (2005) Inheritance of some fertility traits in three successive generations of Holstein Friesian cattle. Arch Tierz 48, 5-11

Lucy MC (2001) Reproductive Loss in High-Producing Dairy Cattle: Where Will It End? J Dairy Sci 84, $1277-93$ 
Manteuffel G (2002) Central nervous regulation of hypothalamic-pituitary-adrenal axis and its impact on fertility, immunity, metabolism and animal welfare - a review. Arch Tierz 45, 575-95

Oschika E (2002) Aktuelle Aspekte der Rinderzucht - Anforderungen an Zuchtorganisation und Vermarktung. Arch Tierz 45 Sonderheft, 45-50

Platen M, Krocker M, Lindemann E, Gross U (1999) Einfluss des Erstkalbealters auf Fruchtbarkeit und Leistung bei Milchkühen. Arch Tierz 42, 417-29

Royal MD, Darwash AO, Flint APF, Webb R, Wooliams JA, Lamming GE (2000) Declining fertility in dairy cattle: changes in traditional and endocrine parameters of fertility. Anim Sci 70, 487-501

Schröder UJ, Staufenbiel R (2003) Konditionsbeurteilung per Ultraschall in der Herdenbetreuung. Teil 2: Rückenfettdicke und Fruchtbarkeit. Tierärztl Praxis 31, 243-7

Seeland G, Henze C (2003) Beziehungen zwischen Milchleistung und Fruchtbarkeit in einer Schwarzbuntpopulation nach intensive Steigerung der Milchleistung. Arch Tierz 46, 103-12

Spain J (2005) Implementing a Nutritional Management Strategy to Enhance Fertility. Proceeding on the Western Canadian Dairy Semina, Kanada

Spiekers H, Potthast V (2004) Erfolgreiche Milchviehfütterung. DLG-Verlag Frankfurt am Main, Deutschland

Staufenbiel R, Schröder U, Gelfert C-C, Panicke L (2003) Körperkondition und Stoffwechselstabilität als Grundlage für eine hohe Milchleistung bei ungestörter Fruchtbarkeit und allgemeiner Gesundheit von Milchkühen. Arch Tierzucht 46, 513-26

Swalve HH (2003) Neue Ansätze in der züchterischen Bearbeitung funktionaler Merkmale. Arch Tierz 46 Sonderheft, 63-71

Tenhagen BA, Rübesam C, Vogel C, Heuwieser W (2005) Leistung Fruchtbarkeit und Gesundheit: Eine komplizierte Dreierbeziehung; Tagungsbeitrag Kongress »Tierschutz Leistung und Gesundheit» Berlin, Deutschland

TLL (2008) Entwicklung der Tierzucht in Thüringen Berichtsjahr 2007. Thüringer Landesanstalt für Landwirtschaft, Jena, Deutschland

TVL (2008) Bericht über Arbeit und Ergebnisse Prüfungsjahr 2007. Thüringer Verband für Leistungs- und Qualitätsprüfungen in der Tierzucht e.V., Erfurt, Deutschland

Varner MA (2002) nach Mahlkow-Nerge K (2008) Hochleistung und Fruchtbarkeit - vereinbar oder nicht? Vortrag zum Milchrindtag 2008 in Götz/Brandenburg, Deutschland

VIT (2008) Jahresabschluss für den Bereich Milchleistungsprüfung für das Kontrolljahr 2006/2007; Vereinigte Informationssysteme Tierhaltung w.V., Verden, Deutschland

Vogel C (2004) Einfluss der Milchleistung und des Laktationsstadiums auf den Besamungserfolg nach Ovulationssynchronisation. Dissertation Freie Universität Berlin, Deutschland

Wangler A, Harms J (2006) Forschungsbericht: Lebensleistung und Nutzungsdauer von Milchkühen. http://www.agrarnet-mv.de/index.php?/content/view/full/1191 [zuletzt gesichtet 17.01.2010]

Zelfel S (2008) Sind hohe Milchleistungen mit guten Reproduktionsergebnissen bei Schwarzbunten Holsteins vereinbar? http://www.portal-rind.de/index.php?name=News\&file=article\&sid=142 [zuletzt gesichtet 17.01.2010]

Corresponding author:

\section{MARTIN WÄHNER}

email: m.waehner@loel.hs-anhalt.de

Hochschule Anhalt (FH), Strenzfelder Allee 28, 06406 Bernburg, Deutschland 